\title{
Resilience as a mediator between conscientiousness and life satisfaction among Chinese college students
}

\author{
Jing Jie \\ Department of Student Affairs \\ Hainan University \\ Haikou 570228, China \\ jiejing@hainu.edu.cn
}

\author{
Jie $\mathrm{Du}$ \\ College of Materials and Chemistry Engineering \\ Hainan University \\ Haikou 570228, China \\ dujie@hainu.edu.cn
}

\begin{abstract}
Conscientiousness is one of predictors of life satisfaction. Resilience plays an important role in conscientiousness. This paper examines the relation between the conscientiousness and life satisfaction, and tests the mediate effect of resilience. We chose 110 undergraduate students from Hainan University to complete a battery of tests, including the conscientiousness subscale of NEO-Five-Factor-Inventory, the resilience scale for Chinese adolescents and the life satisfaction scale for Chinese adolescents. The results show that conscientiousness is significantly positive correlated with life satisfaction and resilience; resilience is significantly positive correlated with general life satisfaction. Regression analyses results reveal that resilience mediates the relation between conscientiousness and life satisfaction.
\end{abstract}

Keywords-resilience; conscientiousness; life satisfaction; personality; college students

\section{INTRODUCTION}

The development of positive psychology has provoked a growing interest in the studies of what constitutes people's happiness and well-being [1,2]. It is well known that high subjective well-being (SWB) is consisting of three components: frequent positive effect, infrequent negative effect, and a global sense of satisfaction with life. The most frequently studied aspects of SWB are life satisfaction and subjective happiness [3].

Life satisfaction is a major component of positive wellbeing, the measure of which may be more stable than measures of affects [4]. Recently, it is of considerable interest to investigate the relationship of SWB with personality $[5,6]$. Numerous researches revealed that an individual's personality could be separated into five distinct dimensions or factors, which is now named as the Five Factor Model of personality. According to this model, individual differences in personality may be described along the factors of conscientiousness, agreeableness, neuroticism, openness to experience, and extraversion [7]. From this point of view, many studies showed that personality is one of the foremost predictors of SWB.

Conscientiousness is one of the five major dimensions in the Big Five model (also called Five Factor Model) of personality. Conscientiousness is related to impulse control. Individuals low on conscientiousness are unable to motivate themselves to perform a task that they would like to accomplish. Previous research found conscientiousness showed a consistent association with higher scores in life satisfaction[8].
However, some evidence showed that personality is related to resilience. While resilience has been defined as resistance to illness, adaptation, and thriving, the ability to bounce back from adversity or recover from stress is closest to its original meaning [9]. Resilience was found to be linked to emotional stability, extraversion, openness to new experiences [10], conscientiousness [10,11, 12] and agreeableness [11, 13].

Like conscientiousness mentioned above, resilience is also related to life satisfaction. The relationship between life satisfaction and resiliency has been studied by some researchers. Fredrickson, et al. found that resilient students' levels of life satisfaction were significantly higher than those of lowresilient students [14]. King concluded that not only resiliency positively related to life satisfaction among individuals experiencing divorce, but also resiliency significantly predicted life satisfaction [15].

Thus conscientiousness and resilience both have relationship with his/her life satisfaction. Although this viewpoint has been identified by many researchers, there are some academic queries still alive. For instance, which factor plays a decisive role for life satisfaction? Does conscientiousness have an indirect influence on life satisfaction? Is the effect of conscientiousness on life satisfaction actually mediated by resilience? To deal with these queries, we explore the relationship between conscientiousness, resilience and life satisfaction for college students. To our knowledge, this is the first study to explore the mediate role of resilience in the relationship between conscientiousness and life satisfaction.

Our hypothesis is that the relationship between conscientiousness and life satisfaction could be explained by resilience. More specifically, although the initial correlation between conscientiousness and life satisfaction is expected to be significant, it is anticipated to drop to a non-significant level once resilience is entered into the regression equation as a mediator. This means that the direct effects model is incomplete, because the effect of conscientiousness on life satisfaction is actually mediated by resilience.

\section{METHOD}

\section{A. Participants}

A total of 110 Hainan University undergraduate students completed a battery of questionnaires. The sample consisted of 
44 males(40\%), 66 females(60\%), ranging in age from 18 to 23 years $(\mathrm{M}=20.72, \mathrm{SD}=0.826)$.

\section{B. Measures}

- The Conscientiousness Subscale of NEO-Five-FactorInventory(Costa and McCrae's, 1992) Conscientiousness was assessed by the Chinese translation of Costa and McCrae's (1992) conscientiousness subscale of NEO-Five-Factor-Inventory. It contains 12 items. The Simplified Chinese Version of NEO-FFI was offered by Psychological Assessment Resource, Inc.

- Resilience Scale for Chinese Adolescents(Hu Y Q and Gan Y Q,2008)[16] The 27 items of the RS(Chinese Version)are organized into five factors(goal planning, help-seeking, family support, affect control, and positive thinking), asking respondents to rate themselves along 27 statements on a 5-point Likert scale $(1=$ totally incorrect, 5 = totally correct)

- Life satisfaction Scale for Chinese Adolescents(Zhang $X \mathrm{G}$ et al.,2004)[17] Life satisfaction was measured by the Life satisfaction Scale for Chinese Adolescents. The scale asks respondents to rate themselves along 36 statements on a 7-point Likert scale ( $1=$ totally incorrect, $7=$ totally correct).

\section{Procedure}

Participants received a questionnaire directly after regular classes. All respondents were informed that participation was voluntary and anonymous and told approximately how much time it would take to complete the questionnaire. All participants completed the questionnaires in a quiet environment with a researcher present. It took about 20 minutes for the participants to fill out the questionnaire.

\section{Data analysis}

All the data were analyzed using SPSS version 18.0.Descriptive analyses correlation analyses were performed on all variables. Logistic regression analyses were performed to find out the effects of resilience on conscientiousness and life satisfaction, controlling for gender, age and so on. The odds ratios and corresponding $95 \%$ confidence intervals were calculated. $\mathrm{P}<0.05$ was considered significant for all tests.

\section{RESULTS}

\section{A. Correlations}

Means and standard deviations, internal consistencies, and intercorrelations are listed in Table 1.
TABLE I. DESCRIPTIVE STATISTICS AND CORRELATIONS BETWEEN MEASURES( $\mathrm{N}=110)$

\begin{tabular}{|c|c|c|c|c|c|}
\hline & M & SD & E & R & LS \\
\hline C & 41.529 & 4.535 & 1 & $0.336^{* * *}$ & $0.307^{* *}$ \\
\hline R & 98.760 & 10.572 & & 1 & $0.651^{* * *}$ \\
\hline LS & 171.420 & 19.301 & & 1 \\
\hline \multicolumn{7}{|c|}{ a. Notes:C $=$ Conscientiousness, $\mathrm{R}=$ Resilience, LS $=$ Life Satisfaction } \\
b. Note:* $\mathrm{p}<.05 ; * * \mathrm{p}<.01 ; * * \mathrm{p}<.001$
\end{tabular}

Conscientiousness correlated strongly with both resilience and life satisfaction $(\mathrm{r}=0.336, \mathrm{p}<0.001$ and $\mathrm{r}=0.307, \mathrm{p}<$ $0.01)$. The correlation between resilience and life satisfaction was significant $(r=0.651, \mathrm{p}<0.001)$.

\section{B. Regression Analyses}

The power of life satisfaction predictors might change with demographic characteristics such as gender, age and so on. Therefore, the present investigation controls the influence of these variables by statistical procedures. The result of the hierarchical regression on life satisfaction is shown in Table 2.

TABLE II. SUMMARY OF HIERARCHICAL REGRESSION ANALYSIS FOR PREDICTING LIFE SATISFACTION $(\mathrm{N}=110)$

\begin{tabular}{|c|c|c|c|c|c|c|}
\hline $\begin{array}{l}\text { dependent } \\
\text { variable }\end{array}$ & $\begin{array}{l}\text { independent } \\
\text { Variable }\end{array}$ & $B$ & $S E B$ & $\beta$ & $t$ & $\begin{array}{l}\text { Total } \\
R 2\end{array}$ \\
\hline \multicolumn{7}{|l|}{ Step 1 } \\
\hline $\begin{array}{l}\text { Life Satis- } \\
\text { faction }\end{array}$ & Conscientiousness & 1.348 & 0.405 & 0.314 & $3.333 * *$ & 0.111 \\
\hline \multicolumn{7}{|l|}{ Step 2} \\
\hline Resilience & Conscientiousness & 0.709 & 0.223 & 0.302 & $3.181 * *$ & 0.097 \\
\hline \multicolumn{7}{|l|}{ Step 3} \\
\hline $\begin{array}{l}\text { Life Satis- } \\
\text { faction }\end{array}$ & Resilience & 1.195 & 0.135 & 0.654 & $8.863 * * *$ & 0.440 \\
\hline \multicolumn{7}{|l|}{ Step 4} \\
\hline $\begin{array}{l}\text { Life Satis- } \\
\text { faction }\end{array}$ & $\begin{array}{l}\text { Conscientiousness } \\
\text { and Resilience }\end{array}$ & $\begin{array}{l}0.549 \\
1.126\end{array}$ & $\begin{array}{l}0.334 \\
0.140\end{array}$ & $\begin{array}{l}0.128 \\
0.616\end{array}$ & $\begin{array}{l}1.646 \\
8.040^{* * *}\end{array}$ & 0.454 \\
\hline
\end{tabular}

The first set of equations regressed conscientiousness variable on life satisfaction. Conscientiousness accounted for a significant amount of variance $(11.1 \%)$ in life satisfaction.

The second set of equations regressed conscientiousness on resilience showed a significant portion of the variance $(9.7 \%)$.

The third series equation regressed resilience on life satisfaction revealed a significant portion of the variance (44\%) in life satisfaction.

The fourth set of equation regressed on conscientiousness and resilience of life satisfaction. The model was significant and accounted for $45.4 \%$ of the variance in life satisfaction. Then we compared the beta weights in the model (i.e. beta weights for conscientiousness alone on life satisfaction and conscientiousness when resilience was also included in the equation). The beta weight of conscientiousness was regressed alone on life satisfaction was 0.314 . When resilience was included in the equation, the resulting beta weight was 0.128 , suggesting that resilience acts as a mediator in the relationship between conscientiousness and life satisfaction. The mediate 
effect accounted for $59.22 \%$ of the total effect between conscientiousness and life satisfaction. Figure 1 depicts the test of the mediating effects of resilience in the relationship between conscientiousness and life satisfaction.

\begin{tabular}{|c|c|c|}
\hline $\begin{array}{c}\text { Conscien- } \\
\text { tiousness }\end{array}$ & $0.314^{* *}$ & Life $\begin{array}{c}\text { Satisfac- } \\
\text { tion }\end{array}$ \\
\hline
\end{tabular}

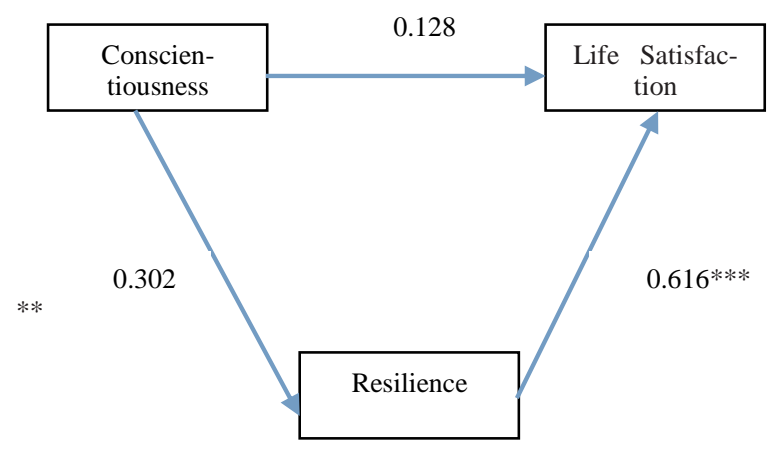

Fig. 1. Path model of the meditational role of resilience in the relation between conscientiousness and life satisfaction. (Note:*p< $0.05 ; * * \mathrm{p}<0.01$; $* * * \mathrm{p}<0.001$.)

\section{DISCUSSION}

The main objective of our study has been to investigate resilience as a mediator of the influence of conscientiousness on life satisfaction.

On a correlational level, the results revealed significantly positive associations between conscientiousness and life satisfaction. This is consistent with the previous research results.

Meanwhile we also found conscientiousness significantly positive correlated with resilience. And the correlation between resilience and life satisfaction was significantly positive. Further results of regression analysis confirmed our hypothesis that the relation between conscientiousness and life satisfaction could be explained by resilience.

The college students scored better resilience, contributing to higher levels of life satisfaction. Moreover, resilient people were found to possess a host of psychological resources, including optimism, tranquility, low neuroticism and high openness. They are more likely to find positive meaning in problems they have to face [14]. These attributes of resilient people were contributed to higher levels of life satisfaction.

Resilience was never treated as the medium variable between the personality and life satisfaction in the past.

Some other reports used the top down approaches to emphasize the role of dispositions or traits in individual differences produced in life satisfaction and other well-being variables [1].Differently, we found people with strong conscientiousness are not only more satisfied with their life, but also higher in resilience which increases their life satisfaction. It means that resilience acts as a mediator between personality and life satisfaction. The results suggest that there is a direct relationship between resilience and life satisfaction, while conscientiousness and life satisfaction are indirect.

The results implied that besides reducing perceived stress, intervention strategies could also focus on cultivation of resilience. The development of resilience is of great value not only to mitigating maladaptive coping and stress, but also to promoting coping mechanisms [18], resulting in higher levels of life satisfaction.

It means that improving resilience lever could increase the life satisfaction degree. The level of resilience can be enhanced through resiliency training program. For example, a 4week resilience education program for college students showed significantly higher resilience scores and more effective coping strategies. Employees at a large medical center increased their resilience over a 12-week period by taking part in a self-directed resiliency training program[19].In addition, adolescents significantly enhanced their resilience through various resilience intervention programs[20,21].

Resiliency training program can also improve undergraduate students' life satisfaction, thus affecting their academic performance and adaptation to university life.

There are still several limitations during this study. Firstly, although resilience appears to play an important role for life satisfaction, we cannot conclude, on the basis of our results, that resilience is the only important variable for life satisfaction. In the current study, resilience was associated with $44 \%$ of the variance for life satisfaction. Resilience cannot be taken as a full explanation of life satisfaction. The findings from the current study should be confirmed by prospective cohort studies in the future. And further study should consider other variation among the effect sizes. Secondly, all data were obtained through self-reported questionnaires, which could introduce response bias. The participants might have underestimated or overestimated the relationship between the study variables. Thirdly, as the sample was only taken from the Hainan Province in southern China, so the results could not be generalized to the people from other areas or with other cultures. Diener(1996) suggested that personality may predict withingroup differences strongly because of the shared environment of that group[22]. So the differences between groups, nations, or cultures(that do not share the same environment), environmental effects are negligible. Some further study are in progress and will be published soon.

\section{REFERENCES}

[1] E. Diener,Subjective well-being,Psychological Bulletin.95,(1984), 542575.

[2] C. R.Snyder, M. E.McCullough,A positive psychology field of dreams: If you build it, they will come,Journal of Social and Clinical Psychology.19,(2000),151-160.

[3] S. Lyubomirsky, Why are some people happier than others? The role of cognitive and motivational processes in well-being,American Psychologist.56, (2001),239-249.

[4] U. Schimmack, S.Oishi, The influence of chronically and temporarily accessible information on life satisfaction judgments,J Pers Soc Psychol. 89,(2005),395-406. 
[5] D. J.Ozer, V. Benet-Martinez,Personality and the prediction of consequential outcomes, Annual Review of Psychology. 57 ,2006) ,401-412.

[6] K. M. DeNeve, H. Cooper,The happy personality: A meta-analysis of 137 personality traits and subjective well-being,Psychological Bulletin. 124,(1998),197-229.

[7] R.R. McCrae, P.T. Jr Costa,Personality trait structure as a human universal, Am Psychol. 52, (1997) ,509-516.

[8] N.Baudin, A.Aluja, J.-P.Rolland, A.Blanch.The role of personality in satisfaction with life and sport. Behavioral Psychology / Psicología Conductual,vol. 19, pp. 333-345,2011

[9] M.M. Fredrickson B.L.Tugade, L F Barrett,Psychological resilience and positive emotional granularity:examining the benefits of positive emotions on coping and health, J Pers. 72, (2004) ,1161-1190.

[10] L. Riolli, V. Savicki, A. Cepani,Resilience in the face of catastrophe:optimism, personality and coping in the Kosovo crisis,J Appl Soc Psychol. 32,(2002),1604-1627.

[11] O. Friborg, D. Barlaug, M. Martinussen, J.H. Rosenvinge, O. Hjemdal,Resilience in relation to personality and intelligence, Int $\mathbf{J}$ Methods Psychiatr Res.14, (2005) ,29-42.

[12] A .Fumham, J. Crump, J. Whelan,Validating the NEO Personality Inventory using assessor's ratings,Pers Individ Dif. 22,(1997),669-675.

[13] M. Davey, D.G. Eaker, L.H. Walters, Resilience processes in adolescents:personality profiles, self-worth, and coping,J Adolesc Res.18, (2003) ,347-362.

[14] B. L. Fredrickson, M. M.Tugade, C. E.Waugh , G. R. Larkin, What good are positive emotions in crisis. A prospective study of resilience and emotions following the terrorist attacks on the United States on September 11th,2001, Journal of Personality and Social gy.84,(2003),365-376.

[15] Information on http://commons.lib.niu.edu/handle/10843/10908

[16] Y.Q.Hu, Y.Q.Gan,Development and Psychometric validity of the Resilience Scale for Chinese Adolescent,Acta Psychologica Sinica. 40,(2008),902-912.

[17] X.G.Zhang,L.G.He,X.Zheng.Adolescent student's life satisfaction:in construct and scale development,Psychological Science.27,(2004),12571260 .

[18] G. Wu, A. Feder, H. Cohen, J.J. Kim, S. Calderon, D.S. Charney, et al. Understanding resilience, Front Behav Neurosci. 7, (2013),10.

[19] V. Sharma, A. Sood, K. Prasad, L. Loehrer, D. Schroeder, B. Brent. Bibliotherapy to decrease stress and anxiety and increase resilience and mindfulness: a pilot trial, Explore (NY). 10,(2014),248-252.

[20] R.K. Hodder, J. Daly, M. Freund, J. Bowman, T. Hazell, J. Wiggers, A school-based resilience intervention to decrease tobacco, alcohol and marijuana use in high school students, BMC Public Health. 11,(2011),722.

[21] S.D. Ritchie, M.J. Wabano, K. Russell, L. Enosse, N.L. Young. Promoting resilience and well-being through an outdoor intervention designed for Aboriginal adolescents, Rural Remote Health. 14,(2014),2523.

[22] E. Diener, C. Diener, Most people are happy,Psychological Science. 7, (1996), 181-185 